\title{
Designing Real Time Business Intelligence Reporting for Better Business Insights
}

\author{
S. Santhosh Baboo, Ph.D \\ Reader, P.G. and Research Dept. of Computer \\ Science D.G.Vaishnav College Chennai 600106
}

\author{
J.Prabhu \\ Research scholar, \\ Computer Science, \\ Manonmaniam Sundaranar University, Tirunelveli, \\ 627012 \\ Assistant Manager, \\ PRICOL Limited
}

\begin{abstract}
Data warehousing has been one of the key implementation practices which was followed in many industries. With constant innovation in hardware and enterprise software's the decision support systems were able to provide more accurate information about the business based on the available historical data. With the rapid change in customer buying habits and frequently changing markets every business needs more insight into all the available data that is in the database. A successful organization needs to take better business decision based on the most recent data. Hence if an analytical reporting tool can provide the business insights based on the real time data available it will definitely help the organizations to understand the business in real time and take better decision on the fly.. The usage of in-memory computing and data storage options like columnar database capability for business intelligence applications can be highly considered for designing the business intelligence systems that provide real time business intelligence reports. This article will analyze the effectiveness of having a real time analytical reporting tool which also use the in-memory technology for business intelligence based applications and see how it can help in making organization to take better business decisions.
\end{abstract}

\section{Keywords}

Real time business intelligence reporting, Performance dashboards, In-Memory analytics.

\section{INTRODUCTION}

The most popular definition for data warehouse is proposed by Bill Inmon as 'subject-oriented', integrated, time-varying, non-volatile collections of data that is used primarily in organizational decision making". Ralph Kimball other guru of Data Warehousing defines a Data Warehouse as [01] "A copy of transaction data, specially restructured for queries and analyses".

Data warehouse has been a key technology for many enterprises to take critical business decisions. Now with the availability of various businesses intelligence tools it helps effectively in better decision making. With the availability of latest hardware innovations like 64 bit RAM and high speed multi core processor help business intelligence reporting to the next level. One question that raise now is "Will it be possible to have real time business intelligence reporting based on the data available in the cube". If this can be achieved the business can respond to the changing business markets and taken the effective business decision in real time. This real time business intelligence can be useful in many industries where the decision making with the frequent changing business trends is a prime factor. With real time business intelligence reporting a company can analyze the company and its position as the business happens in real time

\section{TRADITIONAL DATA WAREHOUSE SYSTEM}

The goal of any data warehouse system is provide analytical reporting based on the historical data available in the cubes that were loaded from multiple source systems. A source system can be of any kind like Customer relationship Management (CRM), Excel based flat files, Logistics and finance application data. An organization which has many legacy systems will use a data warehouse system to consolidate the data from multiple source system and cleanse the data within the business intelligence system and store the data with the business intelligence systems in a common format. It is shown below.

Figure 1: Architecture of traditional Data warehouse system

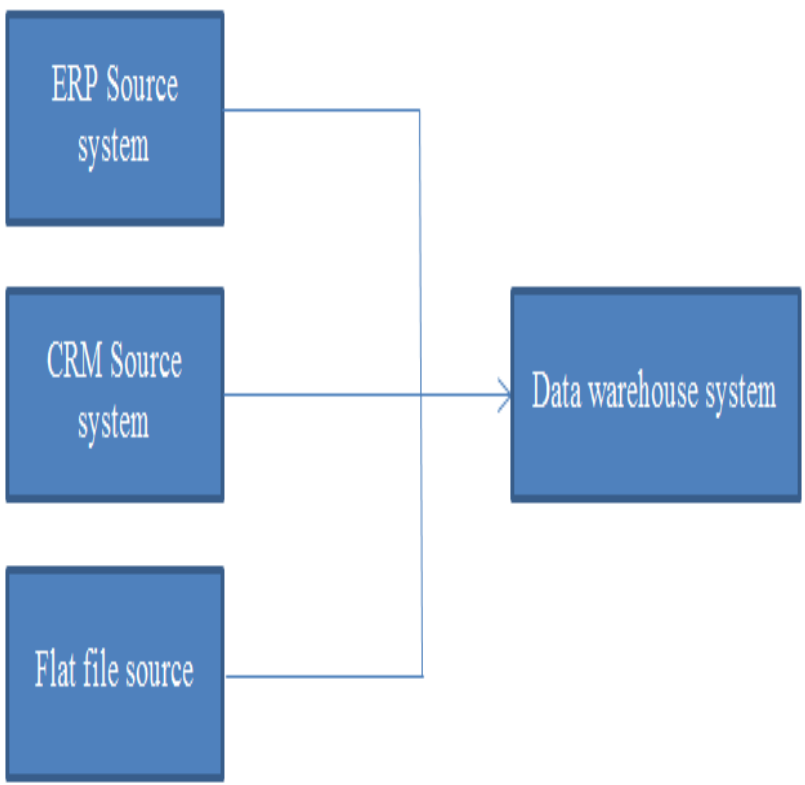

ERP source system can be any OLTP system that stores the transaction data. The real time postings will be done in the 
ERP source system. When a ERP source system need to be connected to Data Warehouse system the transmission method needs to be finalized and ETL tools has to be used.

CRM system is again a system to store the various transaction data related to customer relation management. The data from the CRM system can be used to produce analytical reports and help to understand the customer behavior

Many times a flat file source like MS-EXCEL has to be connected to a Data Warehouse system to send the data for comparison in Data Warehouse system.

Based on the above major three source system we can define the architecture for traditional Data Warehouse system.

The data warehouse system traditionally loads data from various source systems in specific time intervals and the ETL (Extraction Transformation Loading) activities are carried at specific intervals. OLAP systems are usually analyzed as systems that process historical data and provide analytical reports for decision making.

Data consolidation layer captures data from multiple source systems and integrates it into a single persistent target data store, such as a data warehouse. Target data stores contain high-latency data that is typically used for strategic and tactical BI processing. These stores are built using batch data integration applications that pull data from the source systems at predefined scheduled intervals. During data consolidation, data transform techniques may be used to reformat, transform, and cleanse the data. As data changes occur in source systems, change data capture techniques may be used to stream the updates to a target data store. [03] Extract, transformation, and load (ETL) is a technology that supports data consolidation.

\section{REPORTING IN A TRADITIONAL DATA WAREHOUSE SYSTEM}

Within the data warehouse system he data is stored in the cubes which is a multi-dimensional data container. When OLAP systems have to provide analytical reports the data is fetched from the cubes in the data warehouse system. Since the cube is multi-dimensional the report can be viewed with multiple analysis perspective. This is the architecture for a reporting in a traditional data warehouse system. Based on the OLAP reports the organization used to take the business decisions.

Figure 2: Architecture of a data warehouse Analytical reporting

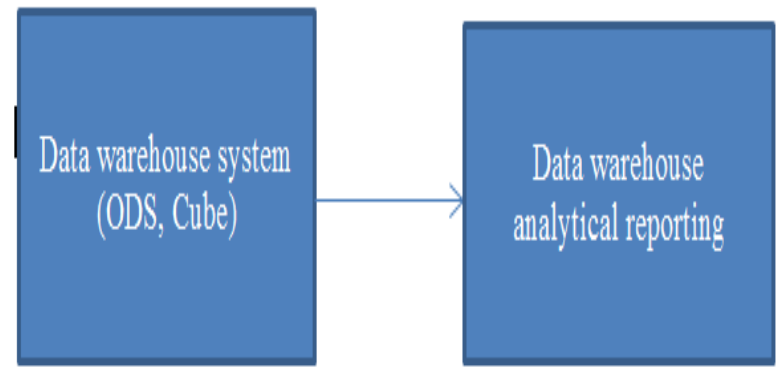

\subsection{Concerns in the current Data warehouse reporting}

The traditional Data warehouse system provide effective reports that help organizations to take effective business decision's but the reports are mostly based on the historical data. A sample report will be like "Overall sales in the north region of the New York city during 2012". This report will help organization to get insights on what happened in the past so that the decisions can be made with that. But the current business markets are changing, the customer buying habits are changing hence an business need to have analytical report that provides data about the business right now, if there is an option to know the real time business behavior the decision made on real time analytical can be more effective.

\section{OPERATIONAL REPORTING}

Operational reports typically provide transactional level or moderately aggregated level of detail. An Open Purchase Order report or number of Open purchase Orders by Buyer is excellent examples of operational reports [04] Operational reporting is designed to support the detailed day-to-day activities of the corporation at the transaction level. In operational reporting, detail is much more important than summary [05]. Many online Transaction Processing systems (OLTP) provide the detailed level of operational reporting.

\section{NEED FOR REAL TIME REPORTING}

Any business will like to be always ahead in the respective market. To stay ahead of the competitors in any market the business need to constantly innovate itself. The business strategy should be able to make the organization better than previous day. The business strategy is always based on the analytical reports or the business data available. In the era of the historical OLAP reporting if the organization were able to understand the customer behavior in the past, now to make the business effective the organizations have to understand the business and customer market in real time that is while the business in running. An retail store should be able to get the information of which products is currently moving better and this can be achieved only if the organization have business intelligence systems that provide real time data. If an retail store can identify the products that move well today at present in real time it can immediately take decision to provide more discounts or other offers to make the particular product to even sell better. This applies to all industries.

OLAP tools enable users to analyze multidimensional data interactively from multiple perspectives. OLAP consists of three basic analytical operations: consolidation (roll-up), drilldown, and slicing and dicing.

The present data warehouse systems have no much usage of dashboards, Dashboards often provide at-a-glance views of KPIs (key performance indicators) relevant to a particular objective or business process (e.g. sales, marketing, human resources, or production).

When we apply the dashboards concept to business a business intelligence dashboard is a data visualization tool that displays the current status of metrics and key performance indicators (KPIs) for an enterprise. Dashboards consolidate and arrange numbers, metrics and sometimes performance scorecards on a single screen. They may be tailored for a specific role and 
display metrics targeted for a single point of view or department. The essential features of a BI dashboard product include a customizable interface and the ability to pull real-time data from multiple sources

\section{PROPOSED SYSTEM ARCHITECTURE}

A real time business intelligence reporting tool needs the data to be fetched from the source system in real time. When the transaction is completed in the system it should be made available in the business intelligence system. Hence a business intelligence system with real time data extraction capability is mandatory in the architecture. When the data gets loaded into the cube the reports that were created based on the cube will be able to use the data and provide analytical reports of the desired format. If this real time reports based alerts have to be sent across multiple destination it will be an added advantage in decision making.

Figure 3: Proposed architecture for real time reporting in business intelligence system (Included Real time reporting)

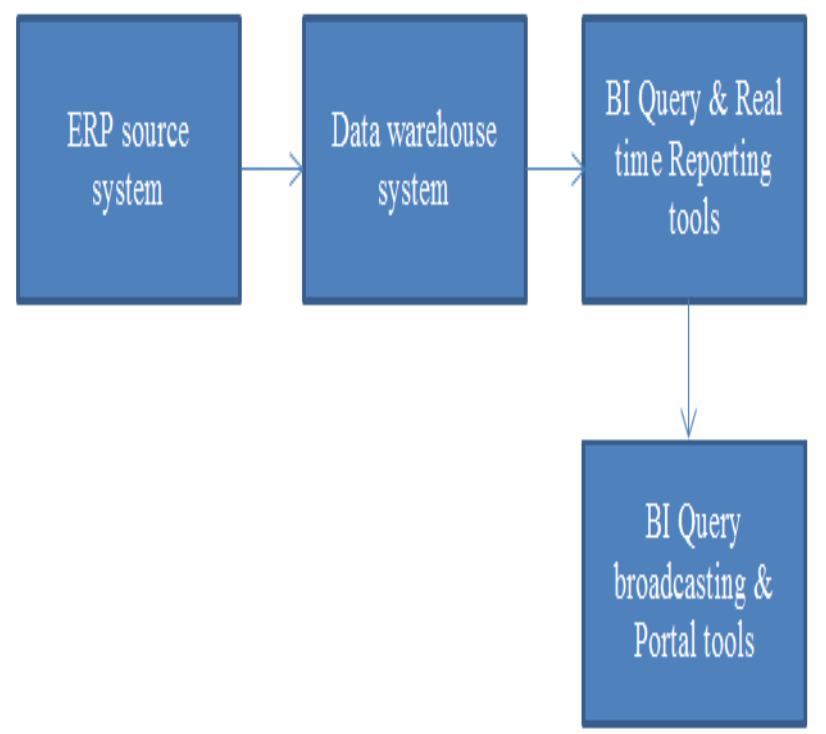

The next figure will show how an In-Memory based business intelligence system reporting can be designed along with mobile and cloud options.
Figure 4: Flow chart for the proposed architecture

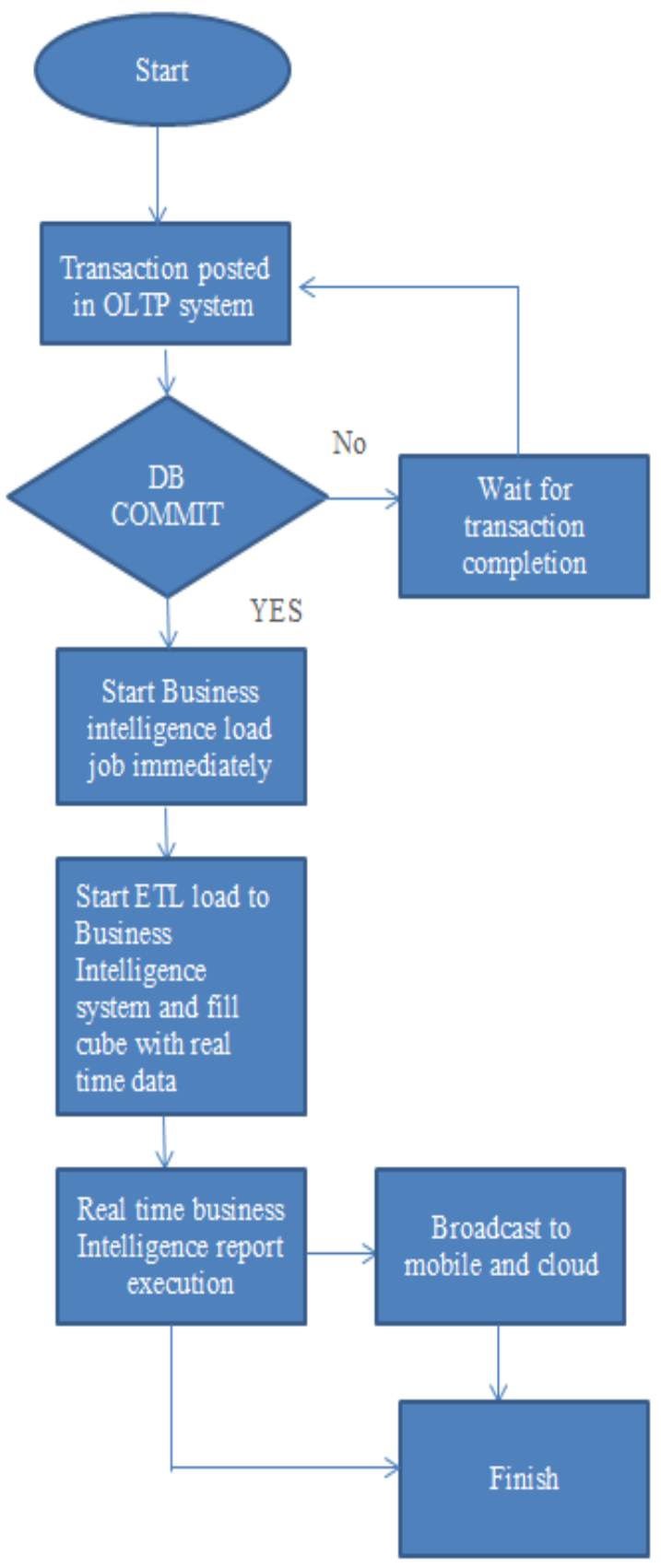

The current innovations in the hardware have led to the usage of In-Memory database for the business intelligence system. The option to use columnar database will help in effective OLAP read operations. When there is effective read operation then the performance of the business intelligence system will be better. The next technology that will capture the future is the availability of cloud computing options. If the results of the real time business intelligence reporting can be made available to the cloud it can be used for the specific purpose. Hence the architecture based on the In-Memory and cloud is suggested below for the design of real time business intelligence system. The broadcasting options and effective dashboards availability in the business intelligence reports make a better clarity for the management to take better business decisions. 
Figure 5: Real time reporting with In-Memory and cloud enabled (Proposed design)

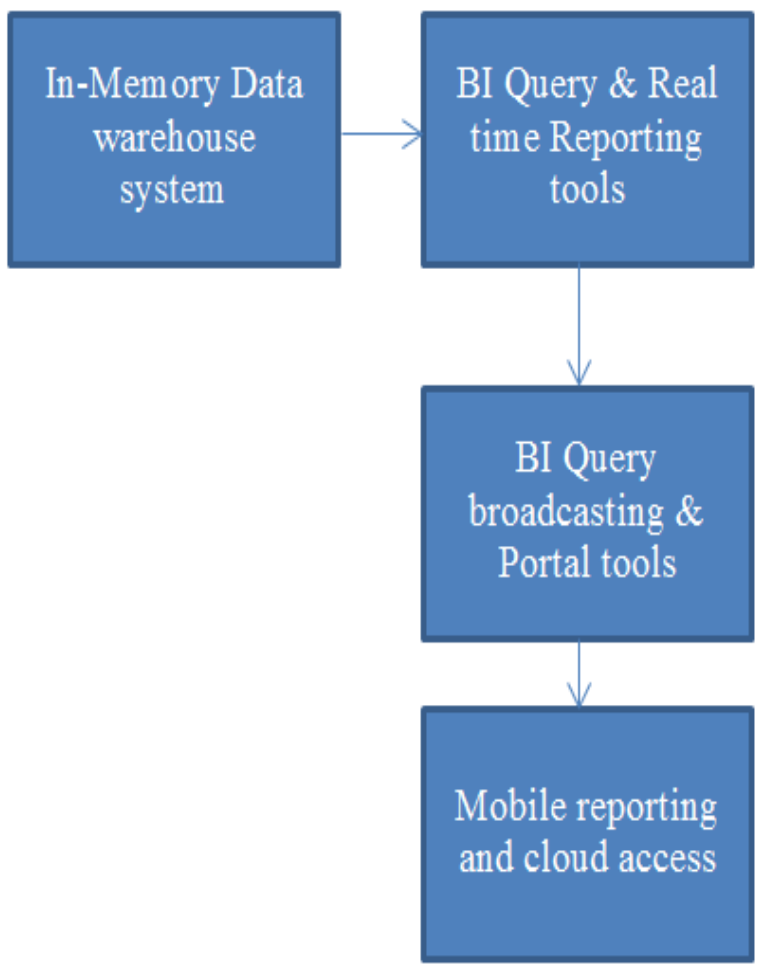

When the reporting is available in the mobile it has to have the option of drill down capability. When a drill down option is expected in a report it has to have many navigational attributes in the Business intelligence system.

\section{DISTINCTIVE FEATURES}

When many Business intelligence tools in the market are analyzed the core distinctive feature that can be seen is OLAP systems based business intelligence reports are still historical in nature. The core distinctive feature that this paper present is the data from ERP system is immediately available in cubes of the business intelligence system

The proposed architecture has many distinctive features

\subsection{Performance consideration}

Business Intelligence system is designed such that the OLTP loads will not cause any issues as in many BI products like SAP BW It is a separate installation. In many real time business intelligence tools the performance is a critical factor as the data has to be available immediately in business intelligence system. The proposed architecture recommend the business intelligence tools to be used with In-Memory and columnar computing options so that there will be no performance issues in business intelligence reporting. The columnar computing option if used will have good read access and hence the reporting performance will be good.

\subsection{Real time alert management}

Alerts are essential for indicating a specific action to be taken from the end user perspective. In business intelligence systems for a report When there is a specific alert maintained for a set of data the real time analytical reports upon finding the specific value will send alert to the predefined users through mail or SMS. This feature will help business to be more aware of the market changing trends.

\subsection{Effective performance dashboards}

As suggested early the usage of the dashboards is becoming more in these days and business needs effective dashboards to suggest the analytical reports effectively. When the real time analytical reports have the effective KPI dashboards along with the charts, graphs and drilldown features with location intelligence analytics it helps to gain better insights.

\subsection{Data visualization}

The age of reports on the tabular format is gone, now business needs more visualized form of data representation. When there is performances degrade the report if it can show the specific one is red alert it can be catchier and adds more value to the report. There are various visualization options available once the data is available for reporting but if the visualization that these papers recommend is to have more chart and graph based features along with drill down the specific entity.

\section{COMPARISION WITH STANDARD DATA WAREHOUSE TOOLS: SAIKU}

When the proposed business intelligence reporting based on In Memory and cloud is compared with other reporting tools like SAIKU a modular open-source analysis suite offering lightweight OLAP which remains easily embeddable, extendable and configurable. The biggest advantage is the usage of In-Memory and the option of having cloud integration. SAIKU do not have the option for the charts and interactive reporting features.

\section{FUTURE WORK}

One of the future researches towards the data warehouse and business intelligence which provide real time reporting is to have predictive analysis features and fetch data from InMemory data warehouse systems and handle big data effectively. The methods to achieve real time data based on the cloud also will be the topic of research in future

\section{CONCLUSION}

This paper discussed about the data warehousing and business intelligence tools that enable the real time business intelligence reporting with various options available now. Customer trends are changing frequently if that can be captured in real time and if the decisions can be taken based on the real time business intelligence reports it can help the organizations to compete in the market better. When data warehouse and business intelligence can use all options available to enable real time reporting it can increase the TCO by assisting in better decision making and help the organization run better. Even many small and medium enterprises will get most out of the data warehouse and real time business intelligence reporting if it can take effective business decision based on the reports available. Business intelligence will no longer have historical reporting and it is 
sure that the future of business intelligence lies in providing the reports and business insights in real time.

\section{REFERENCES}

[1] R. Kimball "The Data Warehouse toolkit", 1996, page 310.

[2] Business intelligence (BI), Nov 2006, http://searchdatamanagement.techtarget.com/definition/b usiness-intelligence

[3] Colin White, BI research , July 2006 The Next Generation of Business Intelligence: Operational BI

[4] Stephen Goldsmith, May 2nd, 2012 http://www.biztech.com/blog/2012/05/operational-vsanalytical-business-intelligence/
[5] Operational and Informational Reporting http://www.informationmanagement.com/issues/20000701/2349-1.html

[6] O'Brien, J. A., \& Marakas, G. M. (2009). Management information systems (9th ed.). Boston, MA: McGrawHill/Irwin

[7] Michael Alexander and John Walkenbach, Excel Dashboards and Reports (Wiley, 2010)

[8] Business intelligence dashboard dhttp://searchbusinessanalytics.techtarget.com/definition/ business-intelligence-dashboard. 